\title{
LOW-DOSE STAVUDINE TRIALS: A PUBLIC HEALTH PRIORITY FOR DEVELOPING COUNTRIES
}

\author{
W D Francois Venter, MB BCh, FCP (SA), Dip HIV Man (SA), DTMぬH (SA), MMed \\ Wits Reproductive Health and HIV Institute (WRHI), Department of Medicine, University of the Witwatersrand and Charlotte \\ Maxeke Johannesburg Academic Hospital, Johannesburg
}

Steve Innes, $M B B C h, M R C P C H, M P h i l$

Mark Cotton, $M B C h B, M M e d, F C P a e d, D T M \ll H, D C H(S A), P h D$

Children's Infectious Diseases Clinical Research Unit (KID-CRU), Tygerberg Children's Hospital and Stellenbosch University, W Cape

The debate around relooking at stavudine dosing, both in terms of the adult low-dose stavudine study and more broadly, is welcome. The study being proposed to evaluate low-dose stavudine v. tenofovir is a fairly standard placebo-controlled non-inferiority study. The study design is not controversial; however, the choice of study drug has attracted critical attention. We have previously discussed the issue of stavudine use, cost and access, and the significant implications of stavudine in developing countries, in detail in a recent article. ${ }^{1}$ We continue to believe that low-dose stavudine clinical trials, in both adults and children, are a priority for developing countries. These studies are being proposed simply because tenofovir is very expensive, and the only available cheaper alternative is stavudine. With recession, unertainty about donor commitments, the compromise of several treatment projects by lack of money, and the plain fact that many developing countries are unable to take ownership of the antiretroviral programme within their budgets, this and similar studies are urgent and necessary for our region to continue to expand access to antiretroviral therapy.

Stavudine was chosen because it is very cheap, easily co-formulated, very well tolerated initially, and requires no laboratory toxicity monitoring in routine clinical practice. Tenofovir was chosen as the comparator for the trial as it is currently the 'gold standard' in many guidelines, and the alternatives (zidovudine (AZT) and abacavir) are now more expensive than tenofovir. Abacavir would be regarded as the equivalent gold standard for paediatric care.

As clinicians involved with antiretroviral (ARV) programmes for the last few years, and having used stavudine in large numbers of both adults and children in the past decade, we identify with the instinctive discomfort of many critics. However, we feel that there is a very strong case for studying stavudine further, and many of the arguments against further trials are not consistent with how we study other antiretrovirals, or indeed medication efficacy in general. Tenofovir certainly has benefits over stavudine: it is dosed daily, and has anti-hepatitis B activity; counter-arguments could be that hepatitis $B$ is easily screened for, and that renal toxicity increasingly recognised with tenofovir has potentially serious consequences in developing countries, where monitoring and access to renal care, dialysis and transplantation are almost nonexistent. ${ }^{2}$ These arguments, however, are likely to be irrelevant when the cost of medication is considered. Broadly, other more substantial arguments fall into the following categories.

\section{STAVUDINE TOXICITY}

This is the most serious disagreement. Critics maintain that stavudine is too toxic to be used, that it is not possible to monitor toxicity safely, and that the trial duration is only 2 years, hence limiting the usefulness of the data.

Toxicity concerns are exclusively based on data around high dosages of stavudine, largely the (now historic) adult $40 \mathrm{mg}$ twice a day (bd) dosage, for which there are extensive toxicity data, including from our own centres. More limited data suggest that $30 \mathrm{mg}$ bd is significantly safer, at least in the medium term, but still carries significant toxicity. We do not contest this - stavudine causes often irreversible and stigmatising lipo-atrophy, as well as peripheral neuropathy and other mitochondrial toxicity in adults. However, all drugs are toxic in sufficient dosages. AZT was originally dosed at far higher levels than currently; toxicity forced the testing of lower doses that were far better tolerated with equal virological suppression levels, and AZT became the standard of first-line care for almost a decade. However, AZT still has some toxicity at this lower dose, and even lower doses are being tried..$^{3-9}$

The original dose-finding studies of stavudine (d4T) were a complex affair, and the originator company did not complete what we consider to have been the natural next phase of study, largely as it was clear that the significant investment on another large clinical trial was unlikely to be recouped, as well as a probable internal assessment that the drug had a significant public relations problem related to lipo-atrophy in developed countries, where profits are made. There is some evidence that dosing at $20 \mathrm{mg}$ bd is safer and gives equivalent virological efficacy, but these data have not been tested in a rigorous manner. ${ }^{5}$

This study plans to repeat those done for drugs such as AZT and many others - optimising the dosage of stavudine so as to minimise toxicity, while maintaining virological efficacy. It also responds to observer calls for more research on stavudine using high-quality trials. $3,5,10$

Critics have maintained that stavudine toxicity is impossible to monitor safely. Safety and toxicity monitoring in the proposed study has been extensively examined by many experts, and we are confident that we can pursue the study safely. Ethics committees are currently examining the proposed toxicity monitoring schedules. 
The doctors involved in the study have plenty of experience in the early recognition of stavudine toxicity.

We agree with critics that the study will not give us evidence on long-term toxicity. Our study is currently funded to run for 2 years, like most registration ARV studies. For many countries, providing 2 years of safe therapy at reduced cost is of significant importance. However, we have extensive lipo-atrophy pre-clinical toxicity monitoring built into the study; if no additional toxicity in the stavudine arm using DEXA scans is demonstrated, it is plausible that the drug could be used for longer, and we therefore intend motivating to extend the study.

Finally, it is contended that this study would never be run in a developed country. We see absolutely no scientific and ethical reasons why not. However, the urgency remains in our region. Rationing has already begun in many programmes, and universal access, with the attendant benefits of HIV prevention suggested by the HPTN 052 trial, is unlikely to be more than a public health dream unless we lower the cost of safe treatment. Even developed countries have had to make rationing decisions, cf. the recent decision to restrict access to first-line ten ofovir in the UK; rationing was made easier by having good data to base these decisions on.

\section{TENOFOVIR COST}

It is asserted that the price of tenofovir is still dropping and will approach that of stavudine. We have consulted extensively over this with the Clinton Health Access Initiative (CHAl) as well as generic companies, and it seems that price equivalence will not be possible, simply because the daily milligram dosage (a daily 300 $\mathrm{mg}$ v. the proposed $40 \mathrm{mg}$ ) is so different, as raw chemicals drive the generic manufacturing costs. Furthermore, the incremental drops in the price of tenofovir (the latest announced by the CHAl in January 2012) are unlikely to be as significant as previous ones, as manufacturing efficiencies have largely been realised.

\section{NEW DRUG AVAILABILITY}

The next assertion is that new drugs are in the offing and will be available by the time this study has results, making the results irrelevant. We believe that confidence in a new drug that will cost-effectively and timeously replace tenofovir is a huge act of faith. Many new medications are indeed being tested, and a small number may show efficacy when this study is completed. However, most drugs fail, even in phase 3 studies, so even this is uncertain. Also, the drugs may not be tested with backbones conventionally used in our context (e.g. raltegravir may be used instead of efavirenz), which may limit agreement on whether we can use the drug safely with available backbone drugs.

Furthermore, the registration process, local regulatory approval, negotiations with generic manufacturers and acceptance into national guidelines mean that it takes many years to go from clinical trial success to broad availability. Tenofovir took over 5 years for registration in South Africa. Prolonged registration is the rule rather than the exception in many developing countries. We may need several more years for adequate costing, price reductions and agreement on priorities for access to this treatment.

We believe that it is responsible to study alternatives to tenofovir and other expensive first-line medications.

\section{OTHER ISSUES}

In paediatric care, abacavir is the current preferred first-line drug owing to concerns about tenofovir toxicity and also to to preserve AZT for a second-line NRTI backbone. Abacavir is very expensive. Stavudine remains the most widely used ARV for HIVinfected children in sub-Saharan Africa. Apart from cost, abacavir has other real problems. Although the incidence of abacavir hypersensitivity reactions is probably low, there is no confirmatory test widely available in the public sector. The HLA-B5701 gene test is simply not available outside large tertiary centres, and the gene appears to be a largely Caucasian one anyway. Nonspecific fever and rash are common in childhood, especially during immune reconstitution, and many children are likely to receive the label 'possible previous abacavir hypersensitivity reaction', which eliminates the option of ever re-introducing abacavir in their regimen. Once abacavir is eliminated, the remaining options in sub-Saharan Africa are zidovudine or stavudine. The danger of zidovudine-related bone marrow suppression (a common problem with varying degrees of severity) is significant and requires some laboratory monitoring. Stavudine offers almost toxicity-free shortterm efficacy. For unknown reasons, thymidine-related peripheral neuropathy has not been documented in pre-pubertal children, and symptomatic lactic acidosis appears remarkably less common than in adults. The incidence of lipo-atrophy is concerning but is strongly dose-related. We think that its frequency and severity will be significantly reduced with the use of low-dose stavudine. In addition, stigmatising lipo-atrophy is avoidable if reasonable awareness is maintained and appropriate drug switches made before lipo-atrophy becomes obvious., ${ }^{9,11}$ An unrecognised benefit of using children's regimens similar to adults' is that it makes children less susceptible to supply-line problems, a huge problem in developing countries. Getting the dose of stavudine right in children is as compelling as it is in adults, and these studies should be prioritised by funders.

In the end, we desperately need alternatives to tenofovir for adults and to abacavir in children in poorer countries. The only current alternative is conventionally dosed stavudine, as AZT and abacavir are more expensive than tenofovir. A minister of health or donor faced with the decision to treat two people with a moderately toxic drug or one with a relatively safe regimen, with the other person definitely dying of AIDS, faces very little choice. Making stavudine safer is an urgent public health issue. We think that doing it safely, efficiently and ethically is possible and should be everyone's priority.

\section{REFERENCES}

1. Innes S, Cotton M, Venter F. Why should we still care about the stavudine dose? Southern African Journal of HIV Medicine 2011:12(4):14-15.

2. Scherzer R, Estrella M, LiY, Deeks SG, Grunfeld C, Shlipak MG. Association of tenofovir exposure with kidney disease risk in HIV infection. AIDS 2012 [Epub ahead of print, 4 Feb]

3. Makinson A, Moing VL, Kouanfack C, Laurent C, Delaporte E. Safety of stavudine in the treatment of HIV infection with a special focus on resource-limited settings. Expert Opin Drug Saf 2008;7(3):283-293.

4. Pujades-Rodríguez M, Dantony E, Pinoges L, et al. AIDS Working Group of Médecins Sans Frontières. Toxicity associated with stavudine dose reduction from 40 to $30 \mathrm{mg}$ in first-line antiretroviral therapy. PLoS One 2011;6(11):e28112 [Epub 21 Nov 2011].

5. Hill A. d4T: keep it or abandon it? Asian Biomedicine 2010;4(4):541-546.

6. Boulle A, Orrell C, Kaplan R, et al. Substitution due to antiretroviral toxicity or contraindication in the first 3 years of antiretroviral therapy in a large South African cohort. Antivir Ther 2007;12:753-760.

7. George JA, Venter WD, Van Deventer HE, Crowther NJ. A longitudinal study of the changes in body fat and metabolic parameters in a South African population of HIV-positive patients receiving an antiretroviral therapeutic regimen containing stavudine. AIDS Res Hum Retroviruses 2009;25(8):771-781.

8. Innes S, Levin L, Cotton M. Lipodystrophy syndrome in HIV-infected children on HAART. South African Journal of HIV Medicine 2009;10(4):76-80.

9. Bobat R, Kindra G, Kiepiela P, et al. Use of abacavir in 30 HIV-infected children from Durban, South Africa: report from a pilot study. Pediatr Infect Dis J 2010;29(9):890.

10. Spaulding A, Rutherford GW, Siegfried N. Stavudine or zidovudine in three-drug combination therapy for initial treatment of HIV infection in antiretroviral-naïve individuals. Cochrane Database Syst Rev 2010 Aug 4; (8):CD008651

11. Eley B. Metabolic complications of antiretroviral therapy in HIV-infected children. Expert Opin Drug Metab Toxicol 2008;4(1):37-49.

12. Rabie H, Henning KL, Schoeman P, De Villiers N, Pretorius GHJ, Cotton M. Abacavir: its use and hypersensitivity. Southern African Journal of HIV Medicine 2009;10(4):81-84. 